\title{
Transnational forest fire fighting: the Malaysian experience
}

\begin{abstract}
The forest fire experiences of Malaysia, Singapore, the Philippines and Thailand in 1997 are reviewed. The problems of transnational co-operation in such cases are examined and the resulting joint lessons are presented.
\end{abstract}

Keyword: Disaster management; Fire; Forestry; Malaysia 